\title{
Cellular Automata for Electrochemistry. Peak Potential Separation Shigeo HAYASHI*
}

\author{
Department of Engineering Science, University of Electro-Communications, \\ 1-5-1 Chofugaoka, Chofu, Tokyo 182-8585, Japan \\ *Corresponding author: hays@pc.uec.ac.jp
}

\section{ABSTRACT}

Electrochemical one-dimensional two-component cellular automata have been tested as to how peak potential separation is related to the parameters corresponding to the electron transfer rate and the sweep rate. Limiting behavior toward $60 \mathrm{mV}$ has been observed, which is consistent with the conventional analytical calculations, and is discussed from the view point of Nernstian limits of the present model.

(c) The Electrochemical Society of Japan, All rights reserved.

Keywords : Cellular Automata, Simulation of E-I Relations, Cyclic Voltammetry, Peak Potential Separation

\section{Introduction}

Cyclic voltammometry, ${ }^{1}$ one of the most popular electrochemical methods, is widely used for the diagnosis of chemical reactions and the analysis of the species participating in the reactions. In a preceding note ${ }^{2}$ (hereafter referred to as I) a kind of one-dimensional cellular automaton (1D-CA) has been proposed as a simplified alternative to the conventional elaborate approach. The 1D-CA, whose primitive form has been known to account for diffusion, ${ }^{3}$ has a unique feature that it is implemented with a reactive reflection wall at the boundary corresponding to the electrode surface. The reactive reflection is characterized by the electron transfer probability, which is a function of the electrode potential.

The 1D-CA does not attempt to solve the electrochemical rate equation but gives a simplified picture of electrochemistry. For example, the diffusion coefficient is not involved explicitly, but the concentration distribution of chemical species is simulated statistically as a function of time. It is possible therefore to envisage electrochemical processes from a microscopic view point, and for this reason 1D-CA is appropriate for pedagogical purposes. However, it is necessary to examine to what extent the 1D-CA parallels with the conventional reaction-diffusion theory of electrochemistry. In this note we thus focus on how the parameters affect voltammograms. In particular, a separation of oxidation and reduction peaks is of our primary interest. According to the theory, ${ }^{4}$ the peak potential separation tends to decrease as the rate of electron transfer increases or the sweep rate decreases, and the lower limit is $60 / n \mathrm{mV}$, where $n$ is the number of electrons transferred for each reaction at the electrode. This behavior will be emulated by the present 1D-CA, although each parameter inevitably has to be varied separately.

\section{Cellular Automaton Model for Electrochemistry}

\subsection{D-CA for electrochemistry}

The methodology of the present note is briefly reviewed. ${ }^{2,5}$ The electrochemical process we consider in the present note is represented by the following quasireversible scheme. ${ }^{6}$

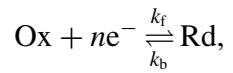

where forward and backward reactions proceed toward equilibrium with rate constants $k_{\mathrm{f}}$ and $k_{\mathrm{b}}$, respectively.
The present 1D-CA consists of the cells interconnected to each other along a straight line between the cathode and anode. The internal state of each cell is represented by the vector $\boldsymbol{P}_{i}^{k}$, which designates the concentration of the two species at time $t=k \delta t$ and position $x=i \delta x$ as

$$
\boldsymbol{P}_{i}^{k}=\left(\begin{array}{c}
P(\mathrm{Ox}) \\
P(\mathrm{Rd})
\end{array}\right)
$$

where $P(\mathrm{Ox})$ and $P(\mathrm{Rd})$ are the concentrations of oxidized $(\mathrm{Ox})$ and reduced (Rd) forms, respectively. The evolution of $\boldsymbol{P}_{i}^{k}(k=0$, $1,2, \ldots$ ) is dictated by Eq. (I-3), i.e., Eq. (3) of $\mathrm{I}$, for $i \geq 2$, by Eq. (I-4) for $i=1$, and by Eq. (I-6) for $i=0$. The last equation, containing the transition probabilities $r_{\mathrm{f}}$ and $r_{\mathrm{b}}$, describes the electrochemical changes at the electrode.

The following relationship has been derived for infinitesimal time changes [cf. Eq. (I-19)],

$$
\frac{r_{\mathrm{f}}}{r_{\mathrm{b}}}=K_{R O} \exp \left(-n \varepsilon / V_{T}\right)
$$

where $V_{T} \approx 25 \mathrm{mV}$ at the room temperature, $\varepsilon$ is the overpotential defined by

$$
\varepsilon=E-E_{\mathrm{eq}}
$$

and a possible existence of the correction term has been ignored.

The parameter $K_{R O}$ is defined by the ratio of the rate constants that appear in Butler-Volmer type equation [cf. Eq. (I-20)]

$$
K_{R O}=\frac{k_{\mathrm{f} 0}}{k_{\mathrm{b} 0}}
$$

The following expressions were proposed in the preceding note [cf. Eqs. (I-21) and (I-22)]

$$
\begin{aligned}
& r_{\mathrm{f}}(\varepsilon)=\frac{v K_{R O}}{1+v K_{R O}} \cdot \frac{1}{1+\exp \left(n \varepsilon / V_{T}\right)} \\
& r_{\mathrm{b}}(\varepsilon)=\frac{v}{1+v K_{R O}} \cdot \frac{1}{1+\exp \left(-n \varepsilon / V_{T}\right)}
\end{aligned}
$$

where a multiplication factor $v$ has been added. As a matter of fact, $k_{\mathrm{f} 0}$ and $k_{\mathrm{b} 0}$ may be identified as the so-called standard rate constant $k_{s}$ divided by $\delta x$. If this is the case, Eq. (5) yields

$$
K_{R O}=1
$$

and then Eqs. (6) and (7) lead to 


$$
r_{\mathrm{f}}(\varepsilon)=r_{\mathrm{b}}(-\varepsilon),
$$

that is, only symmetric cyclic voltammograms will be generated. In order to relax this restriction, we might extend $K_{R O}$ from a constant to a function of $\varepsilon$ such that $K_{R O}(0)=1$ and that $K_{R O}(\varepsilon) \neq 1$ for $\varepsilon \neq 0$. Still easier remedy, however, might be to shift horizontally Eqs. (6) and (7) to make Eq. (3) have the form

$$
\frac{r_{\mathrm{f}}(\varepsilon+\Delta \varepsilon)}{r_{\mathrm{b}}(\varepsilon+\Delta \varepsilon)}=\left[K_{R O} \exp \left(-n \Delta \varepsilon / V_{T}\right)\right] \cdot \exp \left(-n \varepsilon / V_{T}\right)
$$

The first factor in the right-hand side of Eq. (10) can be made to have the value of unity even if $K_{R O}(\varepsilon) \neq 1$. Thus, the equality $r_{\mathrm{f}}=r_{\mathrm{b}}$ can be satisfied at some point displaced from the origin and at the same time asymmetric cyclic voltammograms can be produced. Hereafter, we shall ignore this displacement and accept Eqs. (6) and (7) as valid expressions.

\subsection{Parameters that may affect peak potential separation}

The peak potential separation is known to be determined by the dimensionless parameter involving the rate of electron transfer at the electrode and the rate of potential change, i.e., sweep rate. ${ }^{4,8}$ In the language of 1D-CA the former quantity is directly related to the probability of electron transfer, which is in proportion to $v$. The latter quantity may be affected by the step size in the potential sweeping and the number of iterations at each potential. The following list summarizes the parameters that may affect the peak potential separation [see also Fig. 4(b)].

(i) $\Delta E$ : the step size for incrementing or decrementing the electrode potential $E$, thus related to sweep rate.

(ii) $N_{\text {step }}(\geq 1)$ : the number of iterations for each value of $E$, also related to sweep rate.

(iii) $K_{R O}$ : affects symmetry between oxidation and reduction waves.

(iv) $v\left(\leq 1+1 / K_{R O}\right)$ : controls the rate of electron transfer.

\section{Results and Discussion}

3.1 Peak potential separation and sweep rate: $\Delta E$ and $N_{\text {step }}$

First of all, let us review the results of the conventional theory, which may guide the present discussion: For quasireversible systems the peak potential separation tends to $61 / n \mathrm{mV}$ in the large-value limit of $\psi$ (see also inset of Fig. 3), where ${ }^{4}$

$$
\psi=\frac{k_{s}}{\sqrt{\pi n D v / V_{T}}},
$$

provided that the diffusion coefficients of Ox and Rd have the same value $D$. Here $v$ is the sweep rate. For reversible systems, the peak potential separation is approximately $61 / n \mathrm{mV}{ }^{9}$

The sweep rate should equal the ratio of $\Delta E$ to $N_{\text {step }} \delta t$. Thus, cyclic voltammograms have been calculated for various

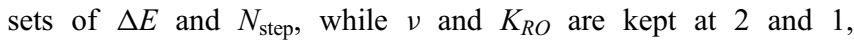
respectively. The results are summarized in Table 1, where peak positions for $\Delta E \geq 0.5 \mathrm{mV}$ are interpolated by fitting to threepoint parabolas. Some fields for small values of $\Delta E$ and large values of $N_{\text {step }}$ have not been calculated since the number of steps, $N_{\text {step }} \times($ spatial range $/ \delta x) \times($ potential range $/ \Delta E)$, becomes impractically too big. The table shows that the same values of $\Delta E / N_{\text {step }}$ do not lead to the same values of the peak separation, but that it increases a little with $\Delta E$. The behavior suggests that the abrupt change in the electrode potential [see also Fig. 4(b)] gives rise to the Cottrel current ${ }^{2}$ and that the sweep rate may be given by

$$
\frac{\Delta E}{N_{\text {step }} \delta t} \rightarrow v
$$

in the limit of small numerator and denominator. This leads to
Table 1. Peak potential separation (in $\mathrm{mV}$ ).

\begin{tabular}{ccccccc}
\hline \multirow{2}{*}{$\begin{array}{c}\Delta E \\
(\mathrm{mV})\end{array}$} & 2 & 4 & 8 & 16 & 32 & 64 \\
\cline { 2 - 7 } & \multicolumn{6}{c}{$N_{\text {step }}$} \\
\hline 0.125 & 61.3 & 60.9 & 60.9 & & & \\
0.25 & 62.8 & 62.3 & 62.0 & & & \\
0.5 & 64.5 & 64.5 & 63.7 & 63.5 & & \\
1 & 67.8 & 66.7 & 66.1 & 66.2 & 66.2 & 66.1 \\
2 & 71.8 & 70.6 & 70.0 & 69.6 & 69.5 & 69.5 \\
5 & 79.7 & 78.0 & 77.0 & 76.5 & 76.3 & 76.2 \\
10 & 88.4 & 86.3 & 86.2 & 84.7 & 84.3 & 84.2 \\
\hline
\end{tabular}

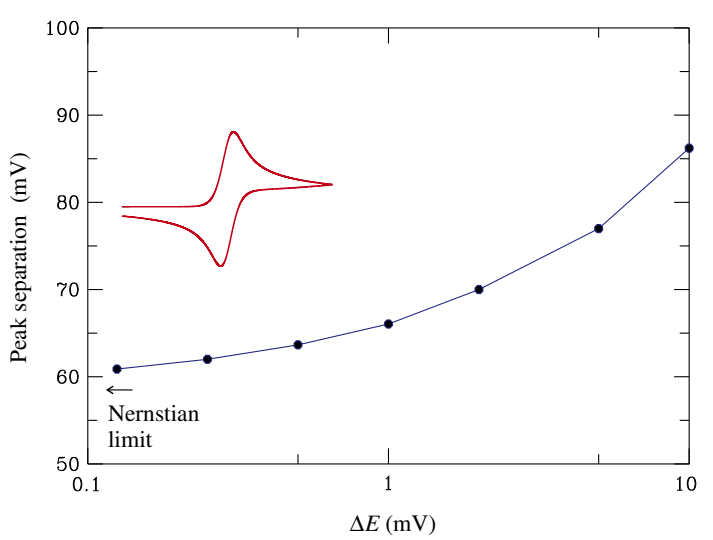

Figure 1. (Color online) Peak potential separation $(\mathrm{mV})$ vs. $\Delta E$ $(\mathrm{mV}) . N_{\text {step }}=8$. Inset is a cyclic voltammogram for $\Delta E=0.125$ $\mathrm{mV}$.

$$
v \propto \frac{1}{N_{\text {step }}}
$$

when the value of $\Delta E$ is fixed. In fact, the peak intensity increases ${ }^{7}$ in proportion to $\frac{1}{\sqrt{N_{\text {step }}}}$, which agrees with the well-known behavior that the intensity increases ${ }^{10}$ with $\sqrt{v}$.

Table 1 shows that the dependence on $N_{\text {step }}$ is of little importance, when other parameters including $\Delta E$ are fixed, but the table shows a stronger dependence on $\Delta E$. In order to see the behavior more clearly, the peak potential separations are plotted in Fig. 1 against $\Delta E$ with $N_{\text {step }}$ and $v$ fixed at 8 and 2, respectively. As the sweep rate is lowered, the peak potential separation decreases and tends to approximately $60 \mathrm{mV}$. The inset shows the cyclic voltammogram for $\Delta E=0.125 \mathrm{mV}$.

\subsection{Peak potential separation and electron-transfer rate: $v$ and $K_{R O}$}

Before calculating cyclic voltammograms, let us see how the electron transfer probabilities vary as a function of $\mathrm{E}$ or $\varepsilon$. $v$ simply changes the probability equally for both of oxidation and reduction. Reduction reactions are enhanced for $K_{R O}>1$ and oxidation for $K_{R O}<1$.

From Eqs. (6) and (7) it follows that

$$
r_{\mathrm{f}}\left(\varepsilon, K_{R O}\right)=r_{\mathrm{b}}\left(-\varepsilon, 1 / K_{R O}\right)
$$

suggesting that the peak separations are the same for $K_{R O}$ and $1 / K_{R O}$. This is really true as shown in Fig. 2, where cyclic voltammograms are shown as insets. The cyclic voltammograms are asymmetric with respect to reduction and oxidation peak heights, but the effects on the peak separation is not as great. Similar behavior was observed for the reaction-diffusion model, where 


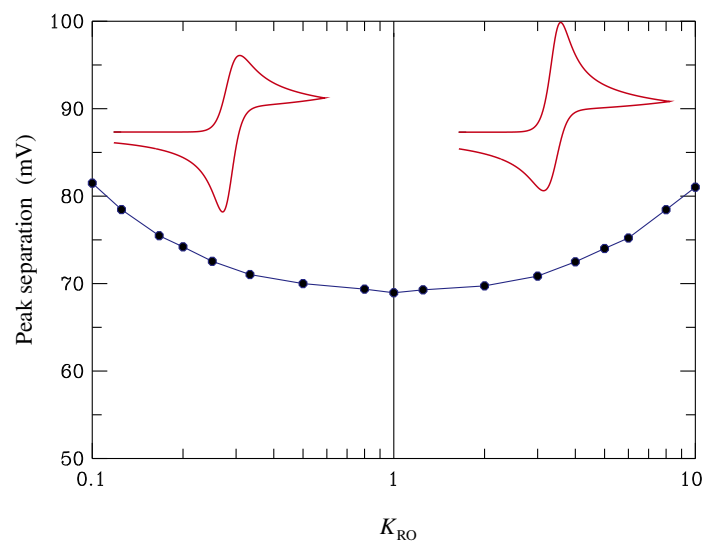

Figure 2. (Color online) Peak potential separation vs. $K_{R O} . v=1$. $N_{\text {step }}=8 . \Delta E=1 \mathrm{mV}$. Cyclic voltammograms at end points, $K_{R O}=1 / 10,10$ are shown as insets.

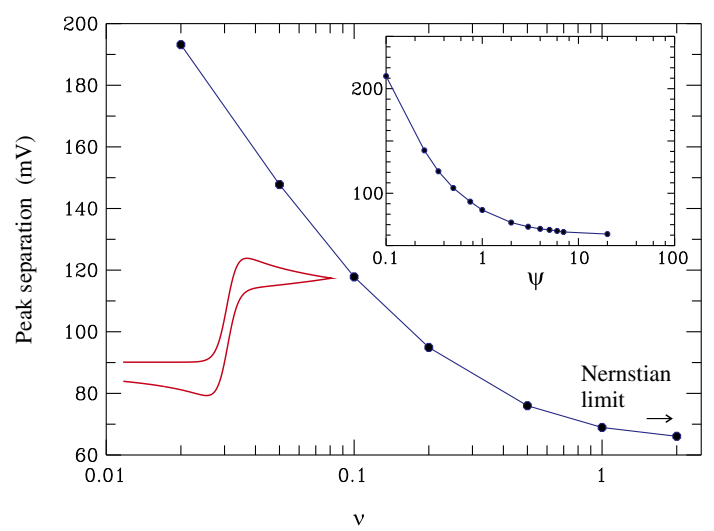

Figure 3. (Color online) Peak potential separation vs. v. $N_{\text {step }}=8$. $\Delta E=1 \mathrm{mV}$. Inset is Nicholson's calculated result. ${ }^{4} \mathrm{~A}$ cyclic voltammogram for $v=0.02$ is also shown.

transfer coefficient $\alpha$ was shown to play a minor role in the peak potential separation. ${ }^{4}$

Instead, the effect of $v$ on the peak separation is more outstanding. Figure 3 shows the dependence of the peak potential separation on $v$ with $\Delta E$ and $N_{\text {step }}$ fixed at $1 \mathrm{mV}$ and 8 , respectively. As the electron transfer rate is lowered, the peak potential separation increases. In the opposite limit, the separation tends to $60 / n \mathrm{mV}$. A cyclic voltammogram for $v=0.02$ is shown as inset, which has the longest separation for the parameters in the graph. The smaller graph shows Nicholson's result ${ }^{4}$ plotted against $\psi$. The present result appears similar to the inset, considering the difference in parameter space, $k_{\mathrm{s}}>0$ and $0<v \leq 1+1 / K_{R O}$.

\subsection{Nernstian limits}

If the electrode potential and the concentrations at the electrode are related by the Nernst relation

$$
E-E_{\mathrm{eq}}=\varepsilon=\frac{V_{T}}{n} \ln \frac{P(\mathrm{Rd})}{P(\mathrm{Ox})},
$$

the system is called reversible or Nernstian. Equation (14) was derived originally for thermodynamical systems, and therefore the electrochemical system must be close to thermodynamical equilibrium. That is, Scheme (1) should be sufficiently fast irrespective of disturbances caused by the change in the electrode potential, and mass transport should be sufficiently fast to satisfy Eq. (14). (a)

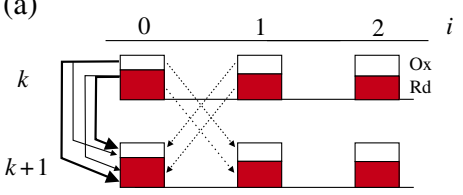

(b)

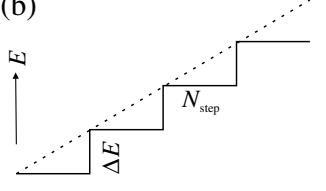

Figure 4. (Color online) (a) One-dimensional two-component cellular automaton making transition at $k$-th time step. Diffusion from $i=1$ is negligible for Nernstian system. (b) The electrode potential changes stepwise.

Let us consider the Nernstian limit for the present 1D-CA. The mass transport is completed in the vertical transitions of Fig. 4(b). During the horizontal $N_{\text {step }}$ cycles, the concentrations are consistent with Eq. (14). The rule for the transition $k \rightarrow(k+1)$ is illustrated in Fig. 4(a). Since $P(\mathrm{Ox})$ and $P(\mathrm{Rd})$ remain unchanged during the transition, we have the following relations

$$
\begin{aligned}
& P(\mathrm{Ox})=P(\mathrm{Rd}) r_{\mathrm{b}}+P(\mathrm{Ox})\left(1-r_{\mathrm{f}}\right) \\
& P(\mathrm{Rd})=P(\mathrm{Ox}) r_{\mathrm{f}}+P(\mathrm{Rd})\left(1-r_{\mathrm{b}}\right)
\end{aligned}
$$

that is,

$$
\frac{r_{\mathrm{f}}}{r_{\mathrm{b}}}=\frac{P(\mathrm{Rd})}{P(\mathrm{Ox})}
$$

and a comparison with Eq. (3) reproduces Eq. (8). However, this condition is naturally insufficient and must be supplemented by another condition that equilibrium should be established quickly. This is made possible if $v$ is close to the maximum. The "Nernstian limits" are marked in Figs. 1 and 3. In these limits, the electron transfer is faster than the disturbances caused by potential sweeping.

\section{Conclusion}

The present 1D-CA has successfully accounted for the changes in the peak potential separation as a function of the parameters corresponding to the rate of electron transfer and the sweep rate, although the conventional reaction-diffusion theory defines a more efficient set of the parameters. ${ }^{4}$ The parameters examined within the present 1D-CA have been the potential step $\Delta E$, the number of steps $N_{\text {step }}$, the asymmetry coefficient $K_{R O}$, and the coefficient for electron transfer probability $v$. The simple methodology should be appropriate for pedagogical purposes such as classroom demonstration and self-learning, wherein the dynamics on the electrode and in the solution over it can be envisaged. The limiting behavior toward $60 / n \mathrm{mV}$ suggests that the present 1D-CA may serve for semi-quantitative purposes.

\section{References}

1. A. J. Bard and L. R. Faulkner, Electrochemical Methods. Fundamentals and Applications, 2nd ed., John Wiley and Sons, New York, NY, p. 226 (2001).

2. S. Hayashi, Electrochemistry, 81, 16 (2013).

3. M. Kac, Selected Papers on Noise and Stochastic Processes (Ed. N. Wax), Dover, New York, NY, p. 295 (1954).

4. R. S. Nicholson, Anal. Chem., 37, 1351 (1965).

5. S. Hayashi, Engineer no Tame no Denki-Kagaku (Electrochemistry for Engineers), Corona-sha, Tokyo, p. 146 (2012). [in Japanese]

6. A. J. Bard and L. R. Faulkner, Electrochemical Methods. Fundamentals and Applications, 2nd ed., p. 94, 191, 236 (2001).

7. S. Hayashi, Engineer no Tame no Denki-Kagaku (Electrochemistry for Engineers), p. 170 (2012). [in Japanese]

8. H. Matsuda and Y. Ayabe, Z. Electrochem., 59, 494 (1955).

9. A. J. Bard and L. R. Faulkner, Electrochemical Methods. Fundamentals and Applications, 2nd ed., p. 242 (2001).

10. A. J. Bard and L. R. Faulkner, Electrochemical Methods. Fundamentals and Applications, 2nd ed., p. 231, 235 (2001). 